\title{
Detection of a redshift 3.04 filament $^{\star}$
}

\author{
P. Møller and J. U. Fynbo
}

\author{
European Southern Observatory, Karl-Schwarzschild-Straße 2, 85748 Garching by München, Germany \\ e-mail: jfynbo@eso.org \\ Received 17 April 2001 / Accepted 26 April 2001
}

\begin{abstract}
The filamentary structure of the early universe has until now only been seen in numerical simulations. Despite this lack of direct observational evidence, the prediction of early filamentary structure formation in a Cold Dark Matter dominated universe has become a paradigm for our understanding of galaxy assembly at high redshifts. Clearly observational confirmation is required. Lyman Break galaxies are too rare to be used as tracers of filaments and we argue that to map out filaments in the high $z$ universe, one will need to identify classes of objects fainter than those currently accessible via the Lyman Break technique. Objects selected via their Ly $\alpha$ emission, and/or as DLA absorbers, populate the faintest accessible part of the high redshift galaxy luminosity function, and as such make up good candidates for objects which will map out high redshift filaments. Here we present the first direct detection of a filament (at $z=3.04$ ) mapped by those classes of objects. The observations are the deepest yet to have been done in Ly $\alpha$ imaging at high redshift, and they reveal a single string of proto-galaxies spanning about $5 \mathrm{Mpc}(20 \mathrm{Mpc}$ comoving). Expanding the cosmological test proposed by Alcock \& Paczyński (1979), we outline how observations of this type can be used to determine $\Omega_{\Lambda}$ at $z=3$.
\end{abstract}

Key words. galaxies: formation - galaxies: high-redshift - quasars: absorption lines - cosmological parameters early Universe - large-scale structure of Universe

\section{Introduction}

For the past three decades, following the first prediction of pancakes by Zel'dovich (1970), simulations have been ahead of the observations when it comes to describing the first structures to form at high redshift. Numerical simulations of structure formation based on the Cold Dark Matter scenario predict that the first large scale structures to form are voids and filaments (Klypin \& Shandarin 1983; White et al. 1987; Evrard et al. 1994; Rauch et al. 1997). In this picture any sightline through the early universe will intersect a large number of voids and filaments, and the variation in the density of neutral hydrogen along the sightline is then observed as the Lyman forest (Petitjean et al. 1995; Theuns et al. 1998). Growth of density fluctuations along the filaments will lead to formation of lumps of cold, self-shielding gas and those are identified, in the simulations, as regions of star formation (Katz et al. 1996; Haehnelt et al. 2000). Because of the high column density of neutral hydrogen in such clouds, they are identified observationally as strong absorbers and the strongest as Damped Ly $\alpha$ Absorbers (DLAs). By poking random sightlines through a virtual universe one may simulate observations, and a given model universe will hence predict a specific correlation between DLA systems and the galaxies hosting the DLAs (Katz et al. 1996). Comparison to real

\footnotetext{
Send offprint requests to: P. Møller, e-mail: pmoller@eso.org

* Based on observations collected at the European Southern Observatory, Paranal, Chile (ESO Programme 64.O-0187).
}

observations of DLA galaxies (Møller \& Warren 1998) has shown that there is very good agreement between observations and simulations. This agreement is encouraging, but it would be of great interest if one could observationally map out the actual high redshift filaments. Apart from being a fundamental test of the validity of the simulations and the structure formation paradigm they represent, knowing the scale size of the filaments at different redshifts will help constrain both cosmological parameters and the mechanisms of galaxy formation.

Unfortunately such a map cannot be constructed via absorption studies, because there is no sufficiently tight mesh of background quasars available. The only way to proceed is to attempt to find enough centers of star formation to map out the filament in its own light, as has been done at low redshift $(z<0.2)$ using galaxy surveys (Gregory \& Thompson 1978; de Lapparent et al. 1991; Bharadwaj et al. 2000). At low redshifts filaments remain, but many of the galaxies have drained into the filament nodes where todays galaxy clusters are formed. In order to identify objects in a high redshift filament one might first try a search for Lyman Break Galaxies (LBGs, Steidel \& Hamilton 1992). Unfortunately only relatively bright galaxies can be found with this technique, and sparse sampling of the filamentary structure does not allow it to be seen easily. However, both DLA galaxies and galaxies selected for their Ly $\alpha$ emission, sample the high redshift galaxy population at much fainter magnitudes than do the LBGs, and should therefore give a better sampling of the 
Table 1. Redshifts and positions of seven Ly $\alpha$ emitters and a Ly $\alpha$ absorber in the field of Q1205-30. The positions are given relative to the quasar coordinates: 12:08:12.7, $-30: 31: 06.10$ (J2000.0). The uncertainty on the redshifts is $0.0012(1 \sigma)$

\begin{tabular}{lrrl}
\hline Object & $\Delta$ RA $(\operatorname{arcsec})$ & $\Delta$ decl. $(\operatorname{arcsec})$ & redshift \\
\hline S7 & $-143.3 \pm 0.6$ & $41.9 \pm 0.2$ & 3.0402 \\
S8 & $-141.5 \pm 0.6$ & $59.7 \pm 0.2$ & 3.0398 \\
S9 & $-124.6 \pm 0.5$ & $63.4 \pm 0.2$ & 3.0350 \\
S10 & $-119.9 \pm 0.5$ & $59.8 \pm 0.2$ & 3.0353 \\
S11 & $-77.8 \pm 0.3$ & $0.9 \pm 0.1$ & 3.0312 \\
S12 & $-43.9 \pm 0.2$ & $54.4 \pm 0.2$ & 3.0333 \\
S13 & $68.3 \pm 0.3$ & $-52.1 \pm 0.2$ & 3.0228 \\
abs & 0.0 & 0.0 & 3.0322 \\
\hline
\end{tabular}

high redshift structure (Fynbo et al. 1999; Haehnelt et al. 2000; Fynbo et al. 2001 (FMT2001)). This has recently been independently confirmed, by deep narrow band Ly $\alpha$ imaging in a field of known LBGs, which reveals about a factor of 6 more candidate Ly $\alpha$ galaxies than LBGs (Steidel et al. 2000). A hint that filamentary structure is indeed present, comes from a few cases where narrow-band Ly $\alpha$ imaging of DLAs was attempted, and one or two additional neighbour Ly $\alpha$ emitters were found. In particular it has been demonstrated that when three DLA and/or Ly $\alpha$ sources are detected in a field, they show a marked tendency to be aligned (Møller \& Warren 1998).

\section{Objects in the field of Q1205-30}

In Fynbo et al. (2000) we reported on a new very deep, $1.1 \times 10^{-17} \mathrm{erg} \mathrm{s}^{-1} \mathrm{~cm}^{-2}(5 \sigma)$, narrow band search for Ly $\alpha$ emitters in a $28 \operatorname{arcmin}^{2}$ field centred on the quasar Q1205-30. The filter was tuned to the wavelength of a strong Ly $\alpha$ absorption line in the quasar spectrum. On March 4-5, 2000 we used the ESO Very Large Telescope on Paranal to obtain spectra of the candidate Ly $\alpha$ emitters in the field. Details of the spectroscopic observations and data reductions have been submitted for publication (FMT2001), but the results are summarised in Table 1 where we list the position and redshift of each of the seven confirmed Ly $\alpha$ emitting objects. In the table we also give the coordinates and redshift of the absorber.

In Fig. 1 we show a sky-coordinate map of the field. While there may here be a hint of an elongated structure running from the SE corner of the CCD frame towards the NW, the statistical evidence for a non-random distribution is weak. However, this may be because we are looking almost along a filament. If this was the case, we would expect the redshifts to change monotonically along the structure suggested by the dotted line in Fig. 1. This should give rise to a strong correlation between redshift and position on the CCD. We performed two tests to assess this correlation, one Monte Carlo and one analytical:

1) For each of the eight objects listed in Table 1 we measured the projected distance on the sky, as well as the difference in redshift, to each of the other seven objects. Those measurements are reproduced in Fig. 2(left). A Spearman correlation test of the data points gives a
$\mathrm{N}$

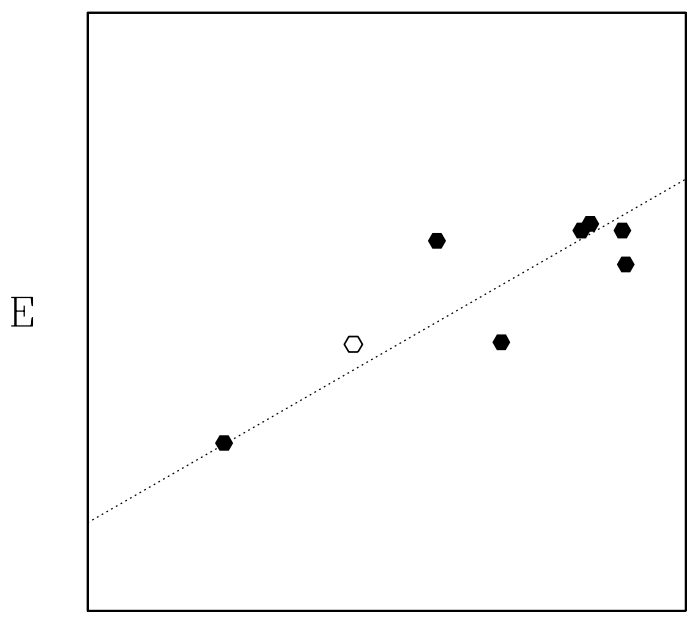

Fig. 1. Position of the eight objects on the CCD. In this and all following figures solid hexagons mark the Ly $\alpha$ emitters, the open hexagon marks the absorber.
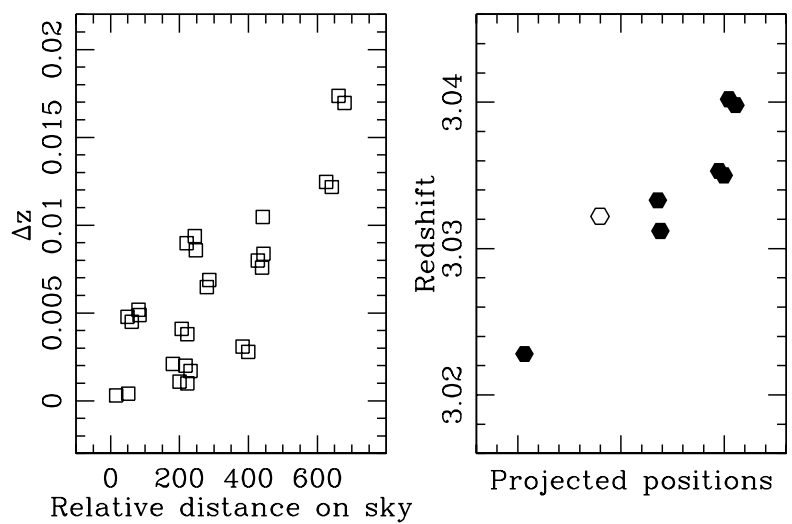

Fig. 2. Left: redshift difference plotted against relative distance on the sky (in CCD pixels). The correlation coefficient is $r_{\mathrm{s}}=0.73$. In random distributions the correlation parameter is worse than that in $99.7 \%$ of all cases. Right: redshift plotted against projected 1D distance (positions projected onto the dotted line in Fig. 1).

correlation coefficient of $r_{\mathrm{s}}=0.73$. We then produced $3 \times 10^{6}$ realizations of a random distribution of eight objects in the observed volume, keeping the position and redshift of the absorber fixed. In only $0.3 \%$ of the simulations did the same test give a similar or better correlation. A more fair comparison is probably to compare against a random distribution with an imposed two-point correlation function as reported by Giavalisco \& Dickinson (2001) $\left(\xi(r)=\left(r / r_{0}\right)^{-\gamma}, r_{0}=1.1 h^{-1} \mathrm{Mpc}, \gamma=2.2\right)$. In this case $0.4 \%$ of the simulations gave a similar or better correlation.

2) Encouraged by this tentative suggestion of a filament, we proceeded to project the position of each object on the CCD onto the line drawn in Fig. 1. In Fig. 2(right) we plot the redshift of each object against the projected position. A Spearman correlation test now gives $r_{\mathrm{s}}=0.881$ for eight objects. In this case we are dealing with statistically independent data points, which confirms the correlation with a probability of $99.82 \%$. 

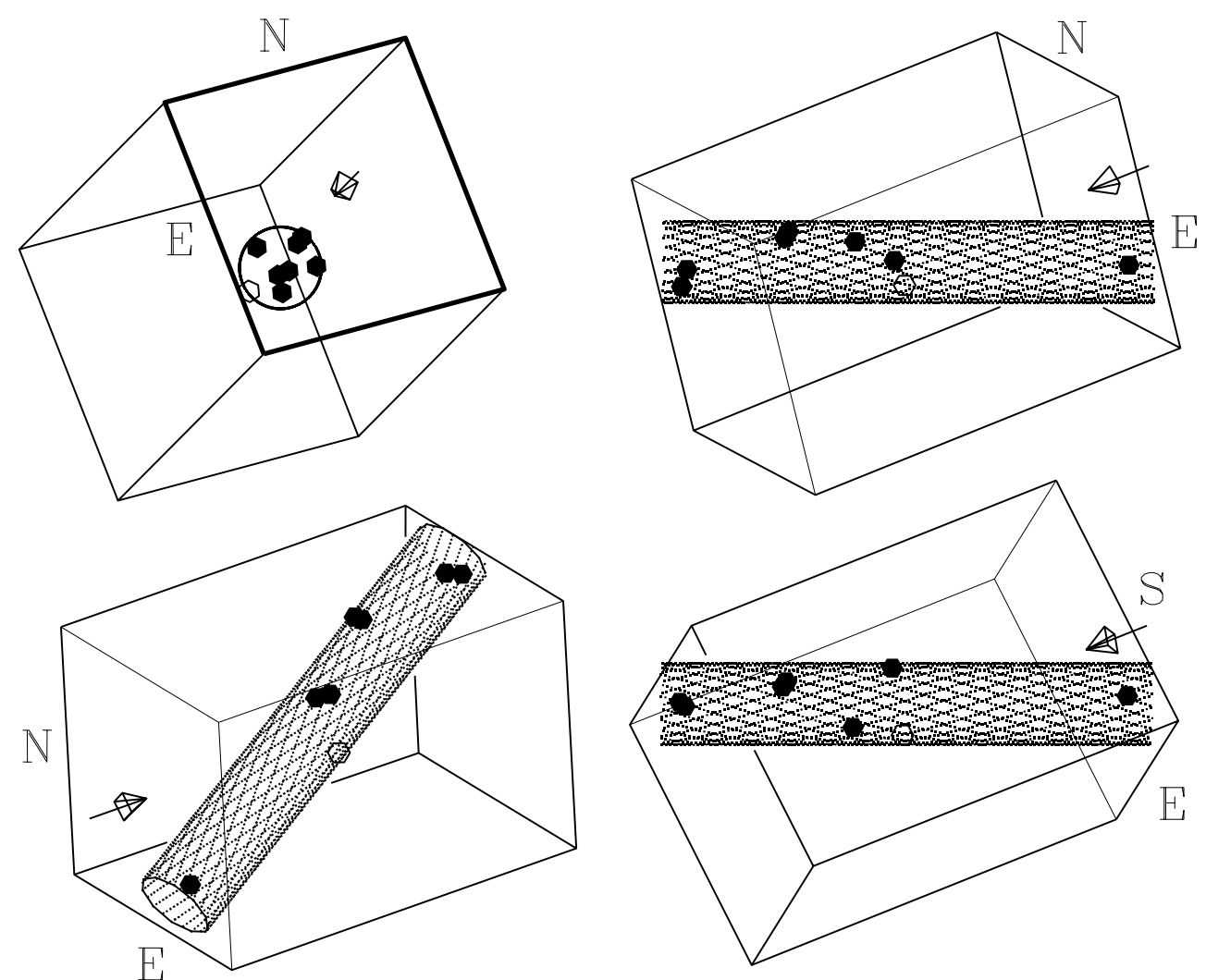

Fig. 3. 3D distribution of the eight objects seen from 4 different viewing-angles. In each of the figures the 3D arrow points in our viewing direction into the sky, and the spiral pattern maps out a cylinder with diameter $400 \mathrm{kpc}$ (see Table 2). The box marks the volume of space observed with our narrow-band Ly $\alpha$ filter. Top left: here we have rotated the view to look along the filament. The thick lines mark the front "entrance window" of the box (corresponding to our CCD image). Top right: the box is here rotated 90 degrees to the right, hence viewing the filament from the left side compared to the end-on view. Bottom right: same as top right but rotated 90 degrees around the filament to give a view of the filament as seen from "above" the view in top left. Bottom left: view from a random angle to give an impression of the 3D structure.

These tests confirm that the eight objects form a tight string-like structure in redshift space. Assuming that the redshifts are caused by Hubble flow, this structure takes the form of a 3D filament similar to those in numerical simulations. The objects are fainter and much more common than the typical LBGs and they presumably represent an earlier evolutionary state than the brighter LBG galaxies. Evidence for this is found in the fact that only $20 \%$ of the LBG population has Ly $\alpha$ in emission, and as a class they show evidence for internal dust absorption suggesting high metallicity (Steidel et al. 2000). In contrast, the numerous faint objects with Ly $\alpha$ in emission have much lower star formation rates than the LBGs and are essentially dust free (FMT2001). In the simulations the final fate of several such objects is to merge into one single galaxy, and for this reason we shall here refer to them as "Ly $\alpha$ Emitting Galaxy-building Objects" (LEGOs).

\section{The Q1205-30 filament at $z=3.04$}

In Fig. 3 we show the 3D structure of the volume of space we are sampling from 4 different viewing-angles. Our sample inside this volume is complete down to a limiting Ly $\alpha$ flux of $1.1 \times 10^{-17} \mathrm{erg} \mathrm{s}^{-1} \mathrm{~cm}^{-2}(5 \sigma)$. The redshift distribution of the LEGOs is consistent with a random distribution when one allows for the selection defined by the filter transmission curve (FMT2001), and we hence conclude that the distribution along the filament is consistent with a random distribution. In other words, we have not detected the ends of the filament, and it may extend further in either direction.

In each of the four figures in Fig. 3 we have enclosed the LEGOs in a hollow "tube" which is meant to illustrate the surface of the filament. The same filament-surface is shown in all the figures. In particular in Fig. 3 (top left) where the filament is viewed from the end, the tube takes the form of a circle. To define the scales of the filament we have chosen three parameters: The length, the radius of the tube and the rms of the projected distribution of the objects around the center of the filament when the filament is viewed from the end. The actual values of those parameters will depend on the chosen cosmology. In Table 2 we list the results for a representative selection of cosmologies. Note that to represent the length we have chosen the largest distance between two detected LEGOs. As discussed above this is in reality a lower limit to the length as we have not yet determined where the endpoints are. The comoving length is hence found to be minimum $20 \mathrm{Mpc}$, in good agreement with recent predictions $\left(20-25 h^{-1}\right.$, Demiański \& Doroshkevich 1999). 
Table 2. Filament parameters for different cosmologies. In all cases have we used $H_{0}=65 \mathrm{~km} \mathrm{~s}^{-1} \mathrm{Mpc}^{-1}$. We defined the length as the distance between the two outhermost LEGOs.

\begin{tabular}{cccc}
\hline Model & rms (kpc) & Radius (kpc) & Length (kpc) \\
\hline$\Omega=0.2, \Omega_{\Lambda}=0.0$ & 280 & 400 & 4400 \\
$\Omega=1.0, \Omega_{\Lambda}=0.0$ & 180 & 260 & 2800 \\
$\Omega=0.3, \Omega_{\Lambda}=0.7$ & 280 & 400 & 4800 \\
$\Omega=0.1, \Omega_{\Lambda}=0.9$ & 390 & 560 & 7700 \\
\hline
\end{tabular}

In an $\Omega=0.2, \Omega_{\Lambda}=0.0$ cosmology the volume sampled by our observations is $30 \mathrm{Mpc}^{3}$. The filament fills only about $7 \%$ of this volume, consistent with a picture of the early universe where the non-linear processes leading to the formation of proto-galaxies are confined within thin filaments.

\section{High $z$ filaments as cosmological probes}

It is interesting to note that five of the seven LEGOs remain undetected in the individual broad-band images (FMT2001), which reach limiting continuum AB magnitudes of $I=26.9$ and $B=27.7$ ( $2 \sigma$ upper limits). Our argument at the outset that searches for LBGs will not detect this type of filament is clearly vindicated. Moreover most of the LEGOs reported here have Ly $\alpha$ fluxes very close to our detection limit. Hence, a slightly shallower Ly $\alpha$ search would likewise have failed to detect the filament. Finally, had the orientation of the filament not been so closely aligned with our line of sight, then only a fraction of the LEGOs would have fallen within the CCD field of view, again making a detection impossible. In this particular case only the fortunate near-alignment and the close-to-critical detection limit made the detection possible. Ly $\alpha$ searches to the same or slightly deeper detection limits but over larger fields should easily pick out several such filaments, thereby making it possible to map the filamentary structure of a significant volume of the early universe.

This opens up an interesting perspective as it will provide an independent way to determine the cosmological constant $\Omega_{\Lambda}$. The conversion of the observed volume into proper coordinates depends on the chosen cosmological model. The effect of using a non-zero $\Omega_{\Lambda}$ is to significantly stretch the volume along the sightline, thereby causing the "length to radius ratio" of filaments to be different for filaments seen from the side and filaments seen end-on. This is a new realisation of the test proposed by Alcock \& Paczyński (1979), who considered the elongation of an idealized spherically symmetric distribution of emitters. In the case of emitters distributed in filaments the test becomes somewhat more complex because of the lack of spherical symmetry, and one will need to consider the statistics of a sample of filaments. At the same time, however, the lack of spherical symmetry offers several independent tests. We consider three tests to be of practical use. First one may consider the distribution of inclination angles of filaments which should be isotropic, secondly the ratio of the rms to the length (as given in Table 2) which must be independent of filament inclination angle, and third the cross-section of the filaments which must in the mean be circular for all inclination angles. In practice one should determine the value of $\Omega_{\Lambda}$ which provides the best fit to all three tests simultaneously. As already pointed out by Alcock \& Paczyński, random motion of objects with respect to the Hubble-flow will add some noise to any such test. We shall return to a detailed discussion of this in a forthcoming paper.

This new set of $\Omega_{\Lambda}$ tests is useful in the redshift range $z=2-4$, is within the capability of current instrumentation and is independent of the successful SN (Riess et al. 1998; Perlmutter et al. 1999) and weak-lensing (Maoli et al. 2001) cosmological tests which currently can be used out to $z \approx 1$. With the complement of large telescopes currently available, we predict rapid progress in this field.

Acknowledgements. We are grateful to B. Thomsen for permission to use results prior to their publication and to S. D. M. White for stimulating discussions and many useful comments on an early draft of this manuscript.

\section{References}

Alcock, C., \& Paczyński, B. 1979, Nature, 281, 358

Bharadwaj, S., Sahni, V., Sathyaprakash, B. S., Shandarin, S. F., \& Yess, C. 2000, ApJ, 528, 21

de Lapparent, V., Geller, M. J., \& Huchra, J. P. 1991, ApJ, 369,273

Demiański, M., \& Doroshkevich, A. G. 1999, MNRAS, 306, 779

Evrard, A. E., Summers, F. J., \& Davis, M. 1994, ApJ, 422, 11

Fynbo, J. U., Møller, P., \& Warren, S. J. 1999, MNRAS, 305, 849

Fynbo, J. U., Thomsen, B., \& Møller, P. 2000, A\&A, 353, 457

Fynbo, J. U., Møller, P., \& Thomsen, B. 2001, A\&A, submitted (FMT2001)

Giavalisco, M., \& Dickinson, M. 2001, ApJ, 550, 177

Gregory, S. A., \& Thompson, L. A. 1978, ApJ, 222, 784

Haehnelt, M. G., Steinmetz, M., \& Rauch, M. 2000, ApJ, 534, 594

Katz, N., Weinberg, D. H., Hernquist, L., \& Miralda-Escudé, J. 1996, ApJ, 457, L57

Klypin, A. A., \& Shandarin, S. F. 1983, MNRAS, 204, 891

Maoli, R., Van Waerbeke, L., Mellier, Y., et al. 2001, A\&A, 368, 766

Møller, P., \& Warren, S. J. 1998, MNRAS, 299, 661

Perlmutter, S., Aldering, G., Goldhaber, G., et al. 1999, ApJ, 517, 565

Petitjean, P., Mueket, J. P., \& Kates, R. E. 1995, A\&A, 295, 9

Rauch, M., Haehnelt, M. G., \& Steinmetz, M. 1997, ApJ, 481, 601

Riess, A. G., Filippenko, A. V., Challis, P., et al. 1998, AJ, 116, 1009

Steidel, C. C., \& Hamilton, D. 1992, AJ, 104, 941

Steidel, C. C., Adelberger, K. L., Shapley, A. E., et al. 2000, ApJ, 532, 170

Theuns, T., Leonard, A., Efstathiou, G., Pearce, F. R., \& Thomas, P. A. 1998, MNRAS, 301, 478

White, S. D. M., Frenk, C. S., Davis, M., \& Efstathiou, G. 1987, ApJ, 313, 505

Zel'dovich, Ya. B. 1970, A\&A, 5, 84 